\title{
An alternative kinematics for multilattices
}

\author{
Mario Pitteri \\ DMMMSA, Università di Padova \\ Via Belzoni 7, 35131 Padova ITALY \\ email: pitteri@dmsa.unipd.it*
}

Dedicated to S. Rionero on the occasion of his $70^{\text {th }}$ birthday

\begin{abstract}
I present an explicit framework for weak phase transformations in complex crystals which is alternative to the one given by Ericksen (2001) and myself (2002b). This approach seems better suited to treat complex lattices with many atoms in the unit cell. As an example, we analyze the model of quartz in Pitteri (2002b) from the present point of view.
\end{abstract}

Keywords Phase transformations, thermoelasticity, crystalline solids, quartz.

\section{Introduction}

The recently renewed interest in the geometry and kinematics of multilattices, aiming at constructing a nonlinear model of the thermomechanical behavior of complex crystals, is acknowledged in Pitteri and Zanzotto (2002), where background for this paper, more details and references are given. There also some still unsolved problems are outlined, in particular the need for a unified kinematics of crystals of different complexity. Here, as in Pitteri (2002a), (2002b), this problem is not addressed, in that only essential multilattices are considered, that is, ones for which the lattice of translations is the maximal one. More work is needed to handle the general case, which is of both theoretical and applicative interest.

The kinematics presented here is a variation of the one in Pitteri (2002b), so here we only describe the differences, addressing the reader to that paper for more details and comments.

${ }^{*}$ This work is part of the research activities of the EU Network 'Phase Transitions in Crystalline Solids', and is partially supported by the Italian M.I.U.R. through the project 'Mathematical Models for Materials Science'. 


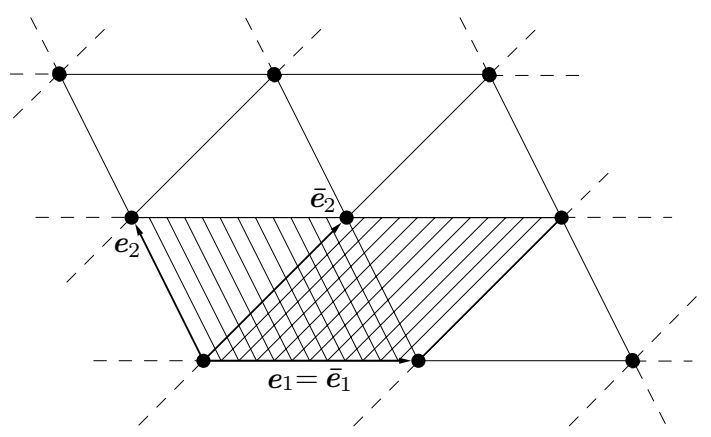

Figure 1: Equivalent bases for a given (planar) lattice

\section{Preliminaries}

As in Pitteri and Zanzotto (2002) I use the summation convention and 'running indices' without specifying their range; for instance in expressions like 'the lattice basis $\boldsymbol{e}_{a}$ ' instead of 'the lattice basis $\left\{\boldsymbol{e}_{a}, a=1,2,3\right\}$ ', or 'the function $\hat{\phi}\left(\boldsymbol{e}_{a}, \boldsymbol{d}_{r}, \theta\right)$ ' instead of 'the function $\hat{\phi}\left(\boldsymbol{e}_{1}, \boldsymbol{e}_{2}, \boldsymbol{e}_{3}, \boldsymbol{d}_{1}, \ldots, \boldsymbol{d}_{n}, \theta\right)$ '. Also, the relations $\leq$ and $<$ between groups mean subgroup of and proper subgroup of, respectively.

Let $\mathbb{Z}$ and $\mathbb{R}$ denote the integral and real numbers, respectively. Consider first the simplest triply-periodic structures, that is, simple lattices (or 1-lattices):

$$
\mathcal{L}=\left\{N^{a} \boldsymbol{e}_{a}, a=1,2,3, N^{a} \in \mathbb{Z}\right\}=\mathcal{L}\left(\boldsymbol{e}_{a}\right) .
$$

The lattice vectors (or lattice basis) $\boldsymbol{e}_{a}$ are linearly independent in $\mathbb{R}^{3}$.

Any basis $\boldsymbol{e}_{a}$ uniquely determines the 1-lattice $\mathcal{L}\left(\boldsymbol{e}_{a}\right)$, but not vice versa:

$$
\mathcal{L}\left(\overline{\boldsymbol{e}}_{a}\right)=\mathcal{L}\left(\boldsymbol{e}_{a}\right) \Leftrightarrow \overline{\boldsymbol{e}}_{a}=m_{a}^{b} \boldsymbol{e}_{b}, \quad m \in G L(3, \mathbb{Z}) ;
$$

here $G L(3, \mathbb{Z})$ is the group of 3 by 3 integral matrices with determinant \pm 1 . The crystallographic point group (or holohedry), $P\left(\boldsymbol{e}_{a}\right)$, of the lattice $\mathcal{L}\left(\boldsymbol{e}_{a}\right)$ is then defined as the group of all the orthogonal transformations mapping $\mathcal{L}\left(\boldsymbol{e}_{a}\right)$ to itself; equivalently:

$$
P\left(\boldsymbol{e}_{a}\right)=\left\{\boldsymbol{Q} \in O(3): \boldsymbol{Q} \boldsymbol{e}_{a}=m_{a}^{b} \boldsymbol{e}_{b}\right\} .
$$

Notice that the basis $\boldsymbol{e}_{a}$ satisfies (3) if and only if the lattice metric

$$
C=\left(C_{a b}\right), \quad C_{a b}=e_{a} \cdot e_{b},
$$

is a fixed point of the map

$$
C \mapsto m^{t} C m .
$$

The conjugacy classes of the holohedries in $O(3)$ correspond to the well known 7 crystal systems. 

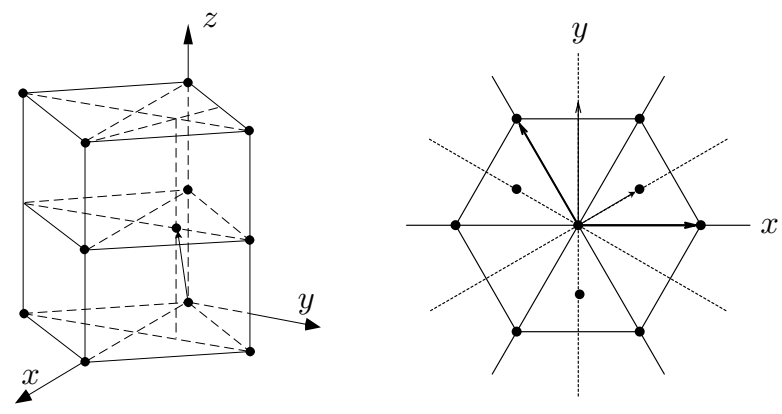

Figure 2: An elementary cell for the hcp lattice

By looking at the right-hand side of the equation defining $P\left(\boldsymbol{e}_{a}\right)$ in (3) we introduce the lattice group $L\left(\boldsymbol{e}_{a}\right)$ of a lattice $\mathcal{L}\left(\boldsymbol{e}_{a}\right)$, associated with its lattice basis $\boldsymbol{e}_{a}$ :

$$
\begin{aligned}
L\left(\boldsymbol{e}_{a}\right) & =\left\{m \in G L(3, \mathbb{Z}): m_{a}^{b} \boldsymbol{e}_{b}=\boldsymbol{Q} \boldsymbol{e}_{a}, \boldsymbol{Q} \in P\left(\boldsymbol{e}_{a}\right)\right\} \\
& =\left\{m \in G L(3, \mathbb{Z}): m^{t} C m=C\right\}
\end{aligned}
$$

By the last equality the lattice group depends on the basis $\boldsymbol{e}_{a}$ only through the corresponding metric $C$, hence can be denoted by $L(C)$. The conjugacy classes of lattice groups in $G L(3, \mathbb{Z})$ correspond to the well known 14 (Bravais) lattice types.

\section{$3 \quad$ Multilattice kinematics}

Real crystals (hexagonal metals, alloys, etc.) are not in general 1-lattices. Their geometry and kinematics can be described by means of multilattices, which are the union of a finite number of nontrivial translates of a 1-lattice. A simple, well known example of 2-lattice is the hexagonal close-packed (hcp) structure, which is sketched in Fig. 2.

In general, an $n$-lattice $\mathcal{M}$ in 3 -dimensional affine space can be defined as follows, in terms of an origin $O$ fixed at will, and in Grassman notation:

$$
\mathcal{M}=\mathcal{M}\left(\boldsymbol{e}_{a}, \boldsymbol{d}_{1}, \ldots, \boldsymbol{d}_{n}\right)=\cup_{r=1}^{n}\left\{O+\mathcal{L}\left(\boldsymbol{e}_{a}\right)+\boldsymbol{d}_{r}\right) ;
$$

$\mathcal{L}\left(\boldsymbol{e}_{a}\right)$ is called the skeletal lattice of $\mathcal{M}$, and each displacement vector $\boldsymbol{d}_{r}$ gives the position, relative to $O$, of a point in one of the simple lattices constituting $\mathcal{M}$. Fig. 3 is a schematic picture of a 2-dimensional (planar) 2-lattice; if the atoms represented by filled circles are phisically indistinguishable from the ones represented by open circles, the 2-lattice is called monatomic, otherwise diatomic.

The multilattice descriptors $\left(\boldsymbol{e}_{a}, \boldsymbol{d}_{r}\right)=: \boldsymbol{\delta}_{\sigma}, \sigma=1, \ldots, n+3$, (in terms of which we can write $\mathcal{M}=\mathcal{M}\left(\boldsymbol{\delta}_{\sigma}\right)$ ) satisfy the following conditions guaranteeing 


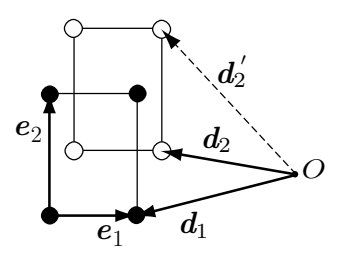

Figure 3: Unit cells of the component 1-lattices of a planar 2-lattice

the three-dimensionality of $\mathcal{M}$ and the non-overlap of the constituent 1-lattices:

$$
e_{1} \cdot e_{2} \times e_{3} \neq 0, \quad d_{r} \neq d_{s}+l_{r s}^{a} e_{a}, \quad r, s=1, \ldots, n, l_{r s}^{a} \in \mathbb{Z} .
$$

An $n$-lattice $\mathcal{M}$ is called essential if its skeletal lattice contains all the translations mapping $\mathcal{M}$ to itself. In this case the lattice cell has minimum volume.

The 'global symmetry group' of essential $n$-lattices expresses the indeterminateness in the choice of the multilattice descriptors:

$$
\mathcal{M}\left(\boldsymbol{\delta}_{\sigma}^{\prime}\right)=\mathcal{M}\left(\boldsymbol{\delta}_{\sigma}\right) \Leftrightarrow \boldsymbol{\delta}_{\sigma}^{\prime}=\nu_{\sigma}^{\tau} \boldsymbol{\delta}_{\sigma}, \quad \nu \in G_{n+3},
$$

where $G_{n+3}<G L(n+3, \mathbb{Z})$ consists of the matrices of the form

$$
\left(\nu_{\sigma}^{\tau}\right)=\left(\begin{array}{ccc|c} 
& m & l_{1}^{a} \cdots l_{n-1}^{a} \\
\hline 0 & 0 & 0 & \\
& \vdots & & \beta \\
0 & 0 & 0 &
\end{array}\right),
$$

for $m \in G L(3, \mathbb{Z}), l_{r}^{a} \in \mathbb{Z}, a=1,2,3, r=1, \ldots, n$, and $\beta=\left(\beta_{r}^{s}\right) \in O(n)$ a permutation matrix of the set $\{1, \ldots, n\}$. Explicitly

$$
\boldsymbol{e}_{a}^{\prime}=m_{a}^{b} \boldsymbol{e}_{b}, \quad \boldsymbol{d}_{r}^{\prime}=\beta_{r}^{s} \boldsymbol{d}_{s}+l_{r}^{a} \boldsymbol{e}_{a}, \quad a, b=1,2,3, r, s=1, \ldots, n .
$$

The group $G_{n+3}$ represents changes of descriptors independently of the chosen origin $O$. Moreover, the subgroup $T_{n+3}$ of skeletal lattice translations, that is, of the matrices $\nu$ for which

$$
m=1 \in G L(3, \mathbb{Z}), \quad \beta=1 \in G L(n, \mathbb{Z}), l_{1}^{a}=l_{2}^{a}=\ldots l_{n}^{a} \in \mathbb{Z}, a=1,2,3,
$$

is a normal subgroup of $G_{n+3}$.

Here and below it is understood that only permutations of physically identical multilattice points must be considered. If we label by an index $\alpha$ the distinct atomic species in the multilattice, $\alpha=1, \ldots, k$, and suitably reorder the component lattices, the permutation matrix $\beta$ becomes a block matrix, the block, say, $\beta_{\alpha}$ being a permutation of atoms of the species $\alpha$.

It is useful to introduce the center of mass $\boldsymbol{g}$ of the displacements, and the barycentric displacements $\tilde{\boldsymbol{d}}_{r}$ :

$$
\boldsymbol{g}=n^{-1} \sum_{r=1}^{n} \boldsymbol{d}_{r}, \quad \tilde{\boldsymbol{d}}_{r}=\boldsymbol{d}_{r}-\boldsymbol{g} .
$$


This and $(11)_{2}$ easily imply, in obvious notation,

$$
\widetilde{\boldsymbol{d}_{r}^{\prime}}=\beta_{r}^{s} \tilde{\boldsymbol{d}}_{s}+\tilde{l}_{r}^{a} \boldsymbol{e}_{a}, \quad \tilde{l}_{r}^{a}=l_{r}^{a}-n^{-1} \sum_{s=1}^{n} l_{s}^{a} \Rightarrow \sum_{s=1}^{n} \tilde{l}_{s}^{a}=0 .
$$

Incidentally the $\tilde{l}_{r}^{a}$ are $1 / n$-integers, unlike the $l_{r}^{a}$ which are integers. It is convenient to denote by $G_{n+3}^{\prime}$ the analogue of $G_{n+3}$ for the integers $l_{r}^{a}$ replaced by the $1 / n$-integers $\tilde{l}_{r}^{a}$ satisfying $(14)_{3}$.

By the above relations two matrices $\nu, \nu^{\prime} \in G_{n+3}$ belong to the same $T_{n+3^{-}}$ coset, which is indicated by $[\nu]\left(=\left[\nu^{\prime}\right]\right) \in G_{n+3} \backslash T_{n+3}$, if and only if they have the same $\tilde{l}_{r}^{a}$, and the same matrices $m$ and $\beta$.

Among all changes of descriptors, particular importance have those which produce an affine isometry of the multilattice onto itself. If, with respect to the chosen origin $O$, we represent the element $e$ of the space $E(3)$ of isometries by a $\operatorname{pair}^{1}(\boldsymbol{t}, \boldsymbol{Q}), \boldsymbol{t} \in \mathbb{R}^{3}, \boldsymbol{Q} \in O(3)$, it must be

$$
\boldsymbol{Q} \boldsymbol{e}_{a}=m_{a}^{b} \boldsymbol{e}_{b}, \quad \boldsymbol{Q} \boldsymbol{d}_{r}+\boldsymbol{t}=\beta_{r}^{s} \boldsymbol{d}_{s}+l_{r}^{a} \boldsymbol{e}_{a} .
$$

The corresponding relations for the center of mass and for the barycentric displacements are

$$
\boldsymbol{Q g}+\boldsymbol{t}=\boldsymbol{g}+n^{-1} \sum_{s=1}^{n} l_{s}^{a} \boldsymbol{e}_{a}, \quad \boldsymbol{Q} \tilde{\boldsymbol{d}}_{r}=\beta_{r}^{s} \tilde{\boldsymbol{d}}_{s}+\tilde{l}_{r}^{a} \boldsymbol{e}_{a} .
$$

In particular, if $O$ coincides with the center of mass, that is, if $\boldsymbol{g}=\mathbf{0}$, then

$$
\boldsymbol{t}=n^{-1} \sum_{r=1}^{n} l_{r}^{a} \boldsymbol{e}_{a} .
$$

For any essentially described multilattice $\mathcal{M}=\mathcal{M}\left(\boldsymbol{\delta}_{\sigma}\right)$ the isometries represented with respect to $O$ by solutions $(\boldsymbol{t}, \boldsymbol{Q})$ of (15) depend on $\mathcal{M}$ itself and neither on its specific descriptors $\boldsymbol{\delta}_{\sigma}$ nor on $O$, and constitute the space group $S(\mathcal{M})<E(3)$ of $\mathcal{M}$. The corresponding matrices $\nu$ form an affinely conjugate (in a suitable sense) 'arithmetic' group, say $A\left(\boldsymbol{\delta}_{\sigma}\right)$, which depends on $\boldsymbol{\delta}_{\sigma}$. Replacing the descriptors $\boldsymbol{\delta}_{\sigma}$ by an equivalent set changes $A\left(\boldsymbol{\delta}_{\sigma}\right)$ to a $G_{n+3}$-conjugate. The orthogonal maps $\boldsymbol{Q}$ appearing in (15) constitute the point group $P(\mathcal{M})$ (or $P\left(\boldsymbol{\delta}_{\sigma}\right)$ ) of $\mathcal{M}$, which is a subgroup of the skeletal holohedry $P\left(\boldsymbol{e}_{a}\right)$; and the quotient $L\left(\boldsymbol{\delta}_{\sigma}\right)=A\left(\boldsymbol{\delta}_{\sigma}\right) \backslash T_{n+3}$, is a finite group isomorphic to $P\left(\boldsymbol{\delta}_{\sigma}\right)$. As for any element of $G_{n+3} \backslash T_{n+3}$, all the matrices $\nu$ representing the same element $[\nu]$ of $L\left(\boldsymbol{\delta}_{\sigma}\right)$ have the same submatrices $m$ and $\beta$, and the same $\tilde{l}_{r}^{a}$.

Assume the origin $O$ to be one of the multilattice points, thus reducing one of the $\boldsymbol{d}_{r}$, say $\boldsymbol{d}_{1}$, to $\mathbf{0}$; and introduce the shifts $\boldsymbol{p}_{r}=\boldsymbol{d}_{r+1}, r=1, \ldots, n-1$, and the $n+2$ multilattice descriptors $\varepsilon_{\sigma}=\left(\boldsymbol{e}_{a}, \boldsymbol{p}_{r}\right)$. The global invariance group $\Gamma_{n+2}<G L(n+2, \mathbb{Z})$ and the lattice group $\Lambda\left(\varepsilon_{\sigma}\right)<\Gamma_{n+2}$ of the multilattice $\mathcal{M}=$ $\mathcal{M}\left(\varepsilon_{\sigma}\right)$, detailed in Pitteri and Zanzotto (2002), are isomorphic to $G_{n+3} \backslash T_{n+3}$ and $L\left(\boldsymbol{\delta}_{\sigma}\right)$, respectively. Therefore properties of the former can be translated into properties of the latter. In particular, the analysis of the invariance of the constitutive equations of a thermoelastic multilattice is considerably simpler if one restricts the attention to suitably small distortions:

\footnotetext{
${ }^{1}$ In Grassmann notation, $\boldsymbol{t}=e(O)-O$, or $e(O)=O+\boldsymbol{t}$.
} 
Proposition 1 Any given set $\boldsymbol{\delta}_{\sigma}$ of multilattice descriptors has a neighborhood $\mathcal{N}$ in $\mathbb{R}^{3(n+3)}$, to be called a (E(3)-invariant) wt-nbhd of $\boldsymbol{\delta}_{\sigma}$, which is invariant under the map $\left(\boldsymbol{e}_{a}, \boldsymbol{d}_{r}\right) \mapsto\left(\boldsymbol{Q} \boldsymbol{e}_{a}, \boldsymbol{Q} \boldsymbol{d}_{r}+\boldsymbol{t}\right)$ for any $\boldsymbol{Q} \in O(3), \boldsymbol{t} \in \mathbb{R}^{3}$, and is such that the transformed $\operatorname{set} \nu(\mathcal{N}):=\left\{\boldsymbol{\delta}_{\sigma}^{\prime}=\nu_{\sigma}^{\tau} \boldsymbol{\delta}_{\tau} \mid \boldsymbol{\delta}_{\sigma} \in \mathcal{N}\right\}$ is disjoint from $\mathcal{N}$ if and only if $\nu$ belongs to $A\left(\boldsymbol{\delta}_{\sigma}\right)$.

Therefore, in any $E(3)$-invariant neighborhood $\mathcal{N}^{\prime}$ of $\boldsymbol{\delta}_{\sigma}$ contained in $\mathcal{N}$ the global $G_{n+3}$-invariance reduces to the invariance under $A\left(\boldsymbol{\delta}_{\sigma}\right)$ :

$$
\text { for any } \overline{\boldsymbol{\delta}}_{\sigma} \in \mathcal{N}^{\prime} \quad A\left(\overline{\boldsymbol{\delta}}_{\sigma}\right) \leq A\left(\boldsymbol{\delta}_{\sigma}\right) .
$$

This result allows us to efficiently reduce the description of the invariance in the wt-nbhds, and to greatly simplify the classification of generic elastic bifurcations for essential multilattices.

For simplicity, in this paper we consider a crystalline solid in equilibrium with a heat-bath of which we only control the temperature. One can extend this treatment to accommodate other controls, for instance pressure, as in Ericksen (2001), or shear stresses, as in Budiansky and Truskinovsky (1993), Simha and Truskinovsky (1996), or Fadda et al. (2002). Here an appropriate thermodynamic potential is the Helmoltz free energy of the multilattice, which is assumed to have a density per unit skeletal cell; this is a sufficiently smooth function

$$
\phi=\hat{\phi}\left(\boldsymbol{e}_{a}, \boldsymbol{d}_{r}, \theta\right)=\bar{\phi}\left(\boldsymbol{\delta}_{\sigma}, \theta\right),
$$

where $\theta$ denotes the absolute environmental temperature, regarded as a control. The free energy density at zero temperature coincides with the internal energy density, and it can be reasonably assumed to depend on the location of the multilattice points also at any given positive temperature. Therefore the functions $\hat{\phi}$ or $\bar{\phi}$ must have the same value on any two equivalent sets of descriptors for the same configuration; hence, for any $\nu \in G_{n+3}$, they satisfy the invariance conditions

$$
\hat{\phi}\left(m_{a}^{b} \boldsymbol{e}_{b}, \beta_{r}^{s} \boldsymbol{d}_{s}+l_{r}^{a} \boldsymbol{e}_{a}, \theta\right)=\hat{\phi}\left(\boldsymbol{e}_{a}, \boldsymbol{d}_{r}, \theta\right), \quad \bar{\phi}\left(\nu_{\sigma}^{\tau} \boldsymbol{\delta}_{\tau}, \theta\right)=\bar{\phi}\left(\boldsymbol{\delta}_{\sigma}, \theta\right),
$$

respectively. In addition, for these functions Galilean invariance reduces to invariance under orientation-preserving affine isometries. In particular

$$
\hat{\phi}\left(\boldsymbol{Q} \boldsymbol{e}_{a}, \boldsymbol{Q} \boldsymbol{d}_{r}+\boldsymbol{t}, \theta\right)=\hat{\phi}\left(\boldsymbol{e}_{a}, \boldsymbol{d}_{r}, \theta\right) \quad \text { for } \quad \boldsymbol{Q} \in S O(3), \boldsymbol{t} \in \mathbb{R}^{3},
$$

hence, by (13),

$$
\begin{gathered}
\hat{\phi}\left(\boldsymbol{e}_{a}, \boldsymbol{d}_{r}, \theta\right)=\hat{\phi}\left(\boldsymbol{e}_{a}, \tilde{\boldsymbol{d}}_{r}, \theta\right)=\Phi\left(\mathrm{s}, C_{a b}, \tilde{d}_{r a}, \theta\right), \quad \text { for } \\
C_{a b}=\boldsymbol{e}_{a} \cdot \boldsymbol{e}_{b}, \quad \tilde{d}_{r a}=\tilde{\boldsymbol{d}}_{r} \cdot \boldsymbol{e}_{a}, \quad \mathrm{~s}=\operatorname{sgn}\left(\boldsymbol{e}_{1} \cdot \boldsymbol{e}_{2} \times \boldsymbol{e}_{3}\right) .
\end{gathered}
$$

As the shifts in Pitteri and Zanzotto (2002), the barycentric displacements $\tilde{\boldsymbol{d}}_{r}$ are multilattice descriptors which are unaffected by any overall translation, hence are a viable choice of variables in the potential which is alternative to the shifts. 
By (14), (22), and the above invariance conditions on $\hat{\phi}$, the function $\Phi$ satisfies the equality

$$
\Phi\left(\mathrm{s}, C_{a b}, \tilde{d}_{r a}, \theta\right)=\Phi\left(\mathrm{ss}(m), m_{a}^{i} C_{i j} m_{b}^{j}, m_{a}^{b}\left(\beta_{r}^{s} \tilde{d}_{s b}+C_{b i} \tilde{l}_{r}^{i}\right), \theta\right)
$$

for any $m \in G L(3, \mathbb{Z})$, any species-preserving permutation $\beta$, and any $1 / n$ integers $\tilde{l}_{r}^{i}$ such that $\sum_{r=1}^{n} \tilde{l}_{r}^{a}=0, a=1,2,3 ; \mathrm{s}(\nu)$ denoting the sign of $\operatorname{det} m(\nu)$. Notice that any such triple $\left(m, \beta, \tilde{l}_{r}^{a}\right)$ forms a matrix $\nu^{\prime} \in G_{n+3}^{\prime}$ and acts according to $(11)_{1},(14)_{1}$ on $\mathbb{R}^{9} \times \mathcal{S}, \mathcal{S} \subset \mathbb{R}^{3 n}$ being the subspace of barycentric displacements.

\section{Phase changes in a wt-nbhd}

Consider a reference configuration of an essential $n$-lattice, described by vectors $\boldsymbol{\delta}_{\sigma}^{0}=\left(\boldsymbol{e}_{a}^{0}, \boldsymbol{d}_{r}^{0}\right)$. The following analogue of (15) holds:

$$
m_{a}^{b} \boldsymbol{e}_{b}^{0}=\boldsymbol{Q} \boldsymbol{e}_{a}^{0}, \quad \beta_{r}^{s} \boldsymbol{d}_{s}^{0}+l_{r}^{a} \boldsymbol{e}_{a}^{0}=\boldsymbol{Q} \boldsymbol{d}_{r}^{0}+\boldsymbol{t},
$$

for any $(\boldsymbol{t}, \boldsymbol{Q})$ representing an element of $S\left(\mathcal{M}\left(\boldsymbol{\delta}_{\sigma}^{0}\right)\right), \nu$ being the corresponding element of $A\left(\boldsymbol{\delta}_{\sigma}^{0}\right)$. Consider also a wt-nbhd $\mathcal{N}$ of $\boldsymbol{\delta}_{\sigma}^{0}$, based on Proposition 1. Since: (1) we restrict the attention to $n$-lattices; (2) our results are local; (3) in the space of descriptors the nonessential multilattices correspond to smooth submanifolds of strictly lower dimension (see Pitteri and Zanzotto (2002)), and (4) $\mathcal{N}$ is the union of disjoint neighborhoods $\mathcal{N}^{+}$and $\mathcal{N}^{-}$of $\boldsymbol{\delta}_{\sigma}^{0}$ and $-\boldsymbol{\delta}_{\sigma}^{0}$, respectively, each one of which is invariant under orientation-preserving affine isometries, we can assume, without loss of generality, that in $\mathcal{N}^{+}$all descriptors $\left(\boldsymbol{e}_{a}, \boldsymbol{d}_{r}\right)$ are essential, and of course $\mathrm{s}=\mathrm{s}^{0}:=\operatorname{sgn}\left(\boldsymbol{e}_{1}^{0} \cdot \boldsymbol{e}_{2}^{0} \times \boldsymbol{e}_{3}^{0}\right)$.

Denoting by $S y m$ the space of symmetric tensors, and by $S m^{>}$the convex cone of the positive definite ones, we normalize the orientation of the (deformed) skeletal lattices of the multilattices in $\mathcal{N}^{+}$by choosing lattice bases of the form $\boldsymbol{e}_{a}=\boldsymbol{U} \boldsymbol{e}_{a}^{0}, \boldsymbol{U} \in$ Sym $^{>}$, and introduce the increments $\boldsymbol{\pi}_{r}\left[\varpi_{r}\right]$ in the referential [barycentric] displacements by means of the following:

$$
\begin{gathered}
\boldsymbol{d}_{r}=\boldsymbol{U}\left(\boldsymbol{d}_{r}^{0}+\boldsymbol{\pi}_{r}\right), \quad \tilde{\boldsymbol{d}}_{r}=\boldsymbol{U}\left(\tilde{\boldsymbol{d}}_{r}^{0}+\varpi_{r}\right), \\
\varpi_{r}=\boldsymbol{\pi}_{r}-n^{-1} \sum_{s=1}^{n} \boldsymbol{\pi}_{s} \Rightarrow \sum_{s=1}^{n} \varpi_{s}=\mathbf{0} .
\end{gathered}
$$

The following is easily proved:

$$
C_{a b}=\boldsymbol{e}_{a}^{0} \cdot \boldsymbol{C} \boldsymbol{e}_{b}^{0}, \quad \boldsymbol{C}=\boldsymbol{U}^{2}, \quad \text { and } \quad \tilde{d}_{r a}=\boldsymbol{e}_{a}^{0} \cdot \boldsymbol{C}\left(\tilde{\boldsymbol{d}}_{r}^{0}+\varpi_{r}\right),
$$

with an obvious analogue for $d_{r a}$ and $\boldsymbol{\pi}_{r}$. Therefore in $\mathcal{N}^{+}$, where $\mathrm{s}$ is fixed,

$$
\hat{\phi}\left(\boldsymbol{e}_{a}, \boldsymbol{d}_{r}, \theta\right)=\hat{\phi}\left(\boldsymbol{e}_{a}, \tilde{\boldsymbol{d}}_{r}, \theta\right)=\Psi\left(\boldsymbol{C}, \boldsymbol{\pi}_{r}, \theta\right)=\Psi\left(\boldsymbol{C}, \varpi_{r}, \theta\right),
$$

for a suitable function $\Psi$. We denote by $P^{+}\left(\boldsymbol{\delta}_{\sigma}^{0}\right)$ the subgroup of positive-determinant elements of $P\left(\delta_{\sigma}^{0}\right)$, and likewise for the $m$ component of the matrices $\nu$ 
in $A^{+}\left(\boldsymbol{\delta}_{\sigma}^{0}\right)<A\left(\boldsymbol{\delta}_{\sigma}^{0}\right)$; and let $L^{+}\left(\boldsymbol{\delta}_{\sigma}^{0}\right)<L\left(\boldsymbol{\delta}_{\sigma}^{0}\right)$ consist of the cosets $[\nu]$ such that $\nu \in A^{+}\left(\boldsymbol{\delta}_{\sigma}^{0}\right)$. For any $\boldsymbol{Q} \in P^{+}\left(\boldsymbol{\delta}_{\sigma}^{0}\right)$ we have

$$
\Psi\left(\boldsymbol{C}, \varpi_{r}, \theta\right)=\Psi\left(\boldsymbol{Q}^{t} \boldsymbol{C} \boldsymbol{Q}, \beta_{r}^{s} \boldsymbol{Q}^{t} \varpi_{s}, \theta\right),
$$

where $\beta$ is the permutation matrix of any $\nu$ satisfying (25) for some $\boldsymbol{t}$ (remember that all such $\nu$ have the same submatrices $m$ and $\beta$ ). Indeed, for any $\nu \in A^{+}\left(\boldsymbol{\delta}_{\sigma}^{0}\right)$ and the corresponding isometry $(\boldsymbol{t}, \boldsymbol{Q})$ given by (15),

$$
\begin{aligned}
\Psi\left(\boldsymbol{C}, \boldsymbol{\pi}_{r}, \theta\right) & =\hat{\phi}\left(\boldsymbol{e}_{a}, \boldsymbol{d}_{r}, \theta\right) \\
& =\hat{\phi}\left(m_{a}^{b} \boldsymbol{e}_{b}, \beta_{r}^{s} \boldsymbol{d}_{s}+l_{r}^{a} \boldsymbol{e}_{a}, \theta\right) \\
& =\hat{\phi}\left(\boldsymbol{U} \boldsymbol{Q} \boldsymbol{e}_{a}^{0}, \boldsymbol{U} \boldsymbol{Q}\left[\boldsymbol{d}_{r}^{0}+\beta_{r}^{s} \boldsymbol{Q}^{t} \boldsymbol{\pi}_{s}+\boldsymbol{Q}^{t} \boldsymbol{t}\right], \theta\right),
\end{aligned}
$$

from which the conclusion follows by (22) and (26)-(29). Notice that the last expression in (31) $)_{3}$ equals $\Psi\left(\boldsymbol{Q}^{t} \boldsymbol{C} \boldsymbol{Q}, \boldsymbol{Q}^{t}\left(\beta_{r}^{s} \boldsymbol{\pi}_{s}+\boldsymbol{t}\right), \theta\right)$, thus providing the extension of (30) to arbitrary reference displacements $\boldsymbol{\pi}_{r}$.

At this point we apply a procedure used by Ericksen (1993) (see also Pitteri and Zanzotto (2002)) to classify generic weak bifurcations in simple lattices: we introduce orthonormal bases $\boldsymbol{V}_{k}, k=1, \ldots, 6$, in Sym and $\boldsymbol{c}_{a}, a=1,2,3$, in $\mathbb{R}^{3}$, and the representations

$$
\boldsymbol{C}=\mathbf{1}+\boldsymbol{E}, \quad \boldsymbol{E}=\sum_{k=1}^{6} y_{k} \boldsymbol{V}_{k}, \quad \boldsymbol{\pi}_{r}=\sum_{a=1}^{3} y_{r}^{a} \boldsymbol{c}_{a},
$$

so that, in particular, $\boldsymbol{C}$ is near $\mathbf{1}$ if and only if the nonlinear strain tensor $\boldsymbol{E} \in$ Sym is near $\mathbf{0}$ or, equivalently, $\left(y_{1}, \ldots, y_{6}\right)$ is near $0 \in \mathbb{R}^{6}$. Here and below we must keep in mind that the coordinates representing the barycentric displacements $\varpi_{r}$ are not all independent because they must satisfy the equations $(34)_{3}$ below. Remember that we have conveniently denoted by $\mathcal{S}$ the $3 n-3$ dimensional linear subspace of $\mathbb{R}^{3 n}$ consisting of barycentric displacements.

In the treatment of the reduced problems in $\S 4$ we will choose the basis $\left(\boldsymbol{c}_{1}, \boldsymbol{c}_{2}, \boldsymbol{c}_{3}\right)$ to coincide with $(\boldsymbol{i}, \boldsymbol{j}, \boldsymbol{k})$ introduced there, and the basis $\boldsymbol{V}_{1}, \ldots, \boldsymbol{V}_{6}$ is meant to be represented in the basis $(\boldsymbol{i}, \boldsymbol{j}, \boldsymbol{k})$ by the matrices in formulae (47)-(49) of Pitteri (2002b). The format chosen here is an alternative to the one proposed there and applied to the $\alpha-\beta$ phase transition in quartz, treated as the 3-lattice consisting of the $\mathrm{Si}$ atoms alone. As is shown below, we obtain here an equivalent description of the possible bifurcations for that 3-lattice.

By putting in a single list $\left(\mathrm{y}_{\mathrm{i}}\right)=\left(y_{1}, \ldots, y_{6}, y_{1}^{a}, \ldots y_{n}^{a}\right)$ the $6+3 n$ coordinates in (32), and denoting by $\tilde{y}_{i}$ the ones related to barycentric displacements (so, $\tilde{y}_{\mathrm{i}} \in \mathbb{R}^{6} \times \mathcal{S}$ ), we can correspondingly introduce a new free energy function $\hat{\Psi}\left(\mathrm{y}_{\mathrm{i}}, \theta\right)=\Psi\left(\boldsymbol{C}, \boldsymbol{\pi}_{r}, \theta\right)$, which then enjoys the invariance

$\hat{\Psi}\left(\mathrm{y}_{\mathrm{i}}, \theta\right)=\hat{\Psi}\left(\overline{\mathrm{y}}_{\mathrm{i}}, \theta\right), \quad \overline{\mathrm{y}}_{\mathrm{i}}=\mathrm{Q}_{\mathrm{ij}} \mathrm{y}_{\mathrm{j}}+\mathrm{t}_{\mathrm{i}}, \quad \mathrm{Q} \in O(6+3 n), \quad \mathrm{t}_{\mathrm{i}}=\left(0, \ldots, 0, \tau^{a}, \tau^{a}, \ldots, \tau^{a}\right)$,

six being the zeros and $n$ the copies of $\tau^{a}=\left(\boldsymbol{Q}^{t} \boldsymbol{t}\right)^{a} \in \mathbb{R}^{3}$. In particular, the affine transformation in $(33)_{2}$ reduces to the linear action by $Q$ on the subspace $\mathbb{R}^{6} \times \mathcal{S}$. By (30) each matrix $Q$ is a block matrix, with a 6 by 6 and a $3 n$ by $3 n$ blocks, each one itself orthogonal. In addition, modulo a suitable reordering of 
the displacements, each atomic species determines itself an orthogonal block in the $3 n$ by $3 n$ submatrix. We denote by $G$ the group of such orthogonal matrices $\mathrm{Q}$ corresponding to elements $[\nu]$ of $L^{+}\left(\boldsymbol{\delta}_{\sigma}^{0}\right)$.

In terms of $\hat{\Psi}$ the equations of equilibrium in $\mathbb{R}^{6} \times \mathcal{S}$, in the absence of loads and in a convenient notation, for $\alpha=1, \ldots, 6, r=1, \ldots, n$, and $a=1,2,3$, are

$$
\hat{\Psi}_{y_{\alpha}}:=\frac{\partial \hat{\Psi}}{\partial y_{\alpha}}\left(\tilde{y}_{i}, \theta\right)=0, \quad \hat{\Psi}_{y_{r}^{a}}\left(\tilde{y}_{i}, \theta\right)+k_{a}=0, \quad \sum_{r=1}^{n} y_{r}^{a}=0
$$

the $k_{a}$ being Lagrange multipliers corresponding to the constraints $(34)_{4}$.

We assume conditions (34) to hold for $\theta=\bar{\theta}$ and $\mathrm{y}_{\mathrm{i}}=0$, the latter giving the (reference) multilattice $\mathcal{M}\left(\boldsymbol{\delta}_{\sigma}^{0}\right)$. Consider now the tensor $L=\left(L_{\mathrm{ij}}\right)=\left(\hat{\Psi}_{\mathrm{y}_{\mathrm{i}} \mathrm{j}}(0, \bar{\theta})\right)$ of moduli at the transition, and the second-order symmetric tensor $\tilde{\mathrm{L}}$ on $\mathbb{R}^{6} \times \mathcal{S}$ associated to the symmetric bilinear form

$$
\left(\left(\tilde{\mathrm{y}}_{\mathrm{i}}\right),\left(\tilde{\mathrm{z}}_{\mathrm{i}}\right)\right) \mapsto \mathrm{L}_{\mathrm{ij}} \tilde{\mathrm{y}}_{\mathrm{i}} \tilde{\mathrm{z}}_{\mathrm{j}}, \quad\left(\tilde{\mathrm{y}}_{\mathrm{i}}\right),\left(\tilde{\mathrm{z}}_{\mathrm{i}}\right) \in \mathbb{R}^{6} \times \mathcal{S} .
$$

If $\tilde{\mathrm{L}}$ is invertible ${ }^{2}$ then, by the implicit function theorem, the equilibrium equations (34) have one solution $\tilde{y}_{i}=\tilde{y}_{i}(\theta)$ in $R^{6} \times \mathcal{S}$ for $\theta$ near $\bar{\theta}$, such that $\tilde{y}_{i}(\bar{\theta})=0$. Also, by continuity and uniqueness, all points on this equilibrium branch have the same symmetry as $\mathcal{M}\left(\boldsymbol{\delta}_{\sigma}^{0}\right)$.

Therefore symmetry breaking can only occur if the tensor $\tilde{L}$ has a nontrivial kernel. The invariance (33) forces, by differentiation, the following identity among the second derivatives of $\hat{\Psi}$ at $(0, \bar{\theta})$ :

$$
L=Q^{t} L Q \text { for any } Q \in G,
$$

hence the eigenspaces of $L$ are invariant under the action of $G$ :

$$
Q^{t} L Q y=L y=\lambda y \quad \Leftrightarrow \quad L Q y=\lambda Q y .
$$

The same is true for the eigenspaces of $\tilde{L}$, each one of which is obtained by intersecting an eigenspace of $L$ with $\mathbb{R}^{6} \times \mathcal{S}$. This fact can be interpreted by saying that invariance forces certain eigenvalues of $L$ (or of $\tilde{L}$ ) to be equal. We restrict the attention to the case, called generic by Ericksen (1993) (see also Pitteri and Zanzotto (2002)), in which the only conditions to be imposed on derivatives of $\hat{\Psi}$ are those guaranteeing that $(0, \bar{\theta})$ is a stable equilibrium at which bifurcation occurs, and those forced by invariance; for instance (36). In particular, the only eigenvalues of $\tilde{L}$ that are equal are the ones that are forced to be so by invariance; or, the eigenspaces of $\tilde{L}$ are irreducible invariant (i.i.) subspaces of $\mathbb{R}^{6} \times \mathcal{S}$ under the action $y \mapsto$ Qy of the group $G$, and exactly one of them is the kernel of $\tilde{L}$. Then, the condition that a stable phase exists, say, for $\theta>\bar{\theta}$ forces all the other eigenvalues to be strictly positive.

We call reduced the action of $\mathrm{G}$ on each i.i. subspace, and also call reduced the group representing such action on that subspace. If one chooses the basis

\footnotetext{
${ }^{2}$ Notice that the kernel of $L$ always contains the nontrivial space $\{0\} \times \mathcal{S}^{\perp}$, where $0 \in \mathbb{R}^{6}$ and $\mathcal{S}^{\perp}$ is the 3 -dimensional orthogonal complement of $\mathcal{S}$ in $\mathbb{R}^{3 n}$.
} 
above aligned with a choice of i.i. subspaces, then each matrix $Q$ is a block matrix, each orthogonal block corresponding to an i.i. subspace, and actually being an element of the reduced group on that subspace.

The fact that the action of $\mathrm{G}$ does not mix the first 6 and the last $3 n$ coordinates, and that the permutation matrices $\beta$ preserve each atomic species (see (30)), implies that the set of i.i. subspaces of $\mathbb{R}^{3} \times \mathcal{S}$ necessarily contain those of either one of the forms

$$
\mathcal{V}_{1} \times\{0\} \quad \text { or } \quad\{0\} \times \mathcal{V}_{2}
$$

where $\mathcal{V}_{1}\left[\mathcal{V}_{2}\right]$ is an i.i. subspace of $\mathbb{R}^{6}$ [of $\mathcal{S}$ consisting in nonvanishing increments of the reference displacements of multilattice points of a single species] and $0 \in \mathcal{S}\left[0 \in \mathbb{R}^{6}\right]$. Case $(38)_{1}$ corresponds to configurational transitions, in which the motif follows the deformation of the skeleton, at least in the beginning. Case $(38)_{2}$ describes structural transitions, which are driven by the deformation of a single species of the motif, followed by a suitable consequent deformation of the other species and of the skeleton. We now follow a classical procedure: we determine the i.i. subspaces of $\mathbb{R}^{6}[$ of $\mathcal{S}]$ for case $(38)_{1}\left[(38)_{2}\right]$, and then consider the corresponding reduced problem; a description of these can be found in Golubitsky et al. (1988), Ericksen (1993), Tolédano and Dmitriev (1996), Pitteri and Zanzotto (2002).

\section{The case of $\beta$-quartz}

At low pressures quartz exhibits two stable phases, called 'low' (or trigonal, or $\alpha$-) quartz and 'high' (or hexagonal, or $\beta$-) quartz; at room pressure, these phases are observed below and above about $574^{\circ} \mathrm{C}$, respectively.

Here we follow James (1987) (and Pitteri and Zanzotto (2002)) by assuming that in any configuration of the $\mathrm{SiO}_{2}$ structure the positions of the $\mathrm{Si}$ atoms be compatible with the definition of a 3-lattice, and neglect the oxigens; thus we describe the crystalline structure of both quartz phases by a monatomic 3-lattice, whose points are the positions of the $\mathrm{Si}$ atoms in the $\mathrm{SiO}_{2}$ lattice. This is a reasonable first choice in the description of generic phase transitions of $\beta$-quartz because, as pointed out above, each transition takes place in an i.i. subspace of displacements of a single atomic species, in this case the one of $\mathrm{Si}$. In the literature the $\alpha-\beta$ transition is attributed to a suitable deformation of tetrahedra having the center at a $\mathrm{Si}$ atom, and the four nearest $\mathrm{O}$ atoms as vertices. This will be analyzed elsewhere, by considering the generic transitions taking place in an i.i. subspace of displacements of the $\mathrm{O}$ atoms.

In both $\alpha$ - and $\beta$-quartz the skeletal lattice type is hexagonal. A common choice of lattice vectors is the following:

$$
e_{1}=(a, 0,0), \quad e_{2}=\left(-\frac{a}{2}, \frac{\sqrt{3} a}{2}, 0\right), \quad e_{3}=(0,0, c)
$$

in an orthonormal basis $(\boldsymbol{i}, \boldsymbol{j}, \boldsymbol{k})$. The rotational subgroup of the corresponding 


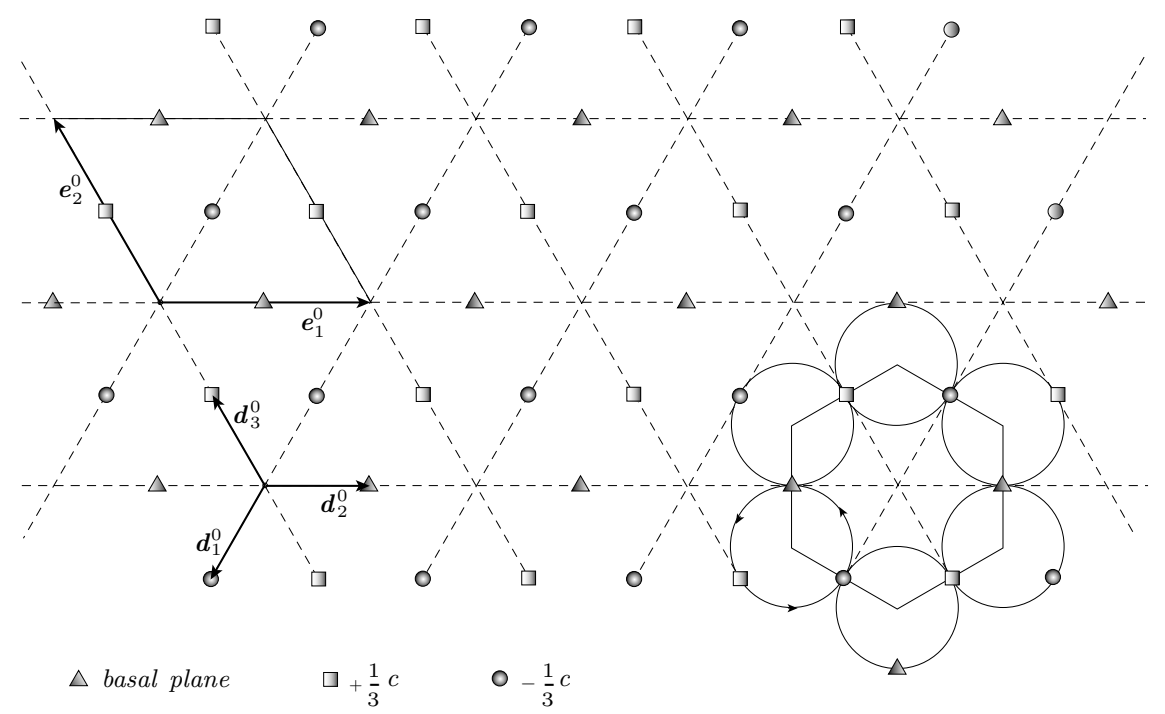

Figure 4: Projection onto the basal plane of the Si atoms in right-handed $\beta$ quartz, and of the descriptors $\boldsymbol{\delta}_{\sigma}^{0}=\left(\boldsymbol{e}_{a}^{0}, \boldsymbol{d}_{1}^{0}, \boldsymbol{d}_{2}^{0}, \boldsymbol{d}_{3}^{0}\right)$ for the 3-lattice given by (39) and (41) with $\lambda>0$

hexagonal holohedry is

$$
\mathcal{H}_{k}=\left\{\mathbf{1}, \boldsymbol{R}_{i}^{\pi}, \boldsymbol{R}_{j}^{\pi}, \boldsymbol{R}_{k}^{\pi}, \boldsymbol{R}_{k}^{\frac{\pi}{3}}, \boldsymbol{R}_{k}^{\frac{2 \pi}{3}}, \boldsymbol{R}_{k}^{\frac{4 \pi}{3}}, \boldsymbol{R}_{k}^{\frac{5 \pi}{3}}, \boldsymbol{R}_{i \pm \sqrt{3} j}^{\pi}, \boldsymbol{R}_{\sqrt{3} i \pm j}^{\pi}\right\}
$$

where $\boldsymbol{R}_{\boldsymbol{v}}^{\omega}$ denotes the rotation by the angle $\omega$ about the direction of the vector $\boldsymbol{v}$. In the crystallographic literature the plane of $\boldsymbol{e}_{1}$ and $\boldsymbol{e}_{2}$ is called the basal plane, and the direction of $\boldsymbol{e}_{3}$ (and of $\boldsymbol{k}$ ) is called the (hexagonal) optic axis.

One of the two possible (enantiomorphic) 3-lattice structures of Si atoms in $\beta$-quartz at the transition temperature $\bar{\theta}$ has descriptors $\boldsymbol{\delta}_{\sigma}^{0}, \sigma=1, \ldots, 6$, where the lattice vectors $\boldsymbol{e}_{a}^{0}$ are given by (39) for suitable choices $a_{0}, c_{0}$, of $a$ and $c$, and barycentric displacements can be chosen as follows:

$$
d_{1}^{0}=-\frac{1}{2}\left(e_{1}^{0}+e_{2}^{0}\right)-\frac{1}{3} e_{3}^{0}, \quad d_{2}^{0}=\frac{1}{2} e_{1}^{0}, \quad d_{3}^{0}=\frac{1}{2} e_{2}^{0}+\frac{1}{3} e_{3}^{0} .
$$

Fig. 4 shows the projection of the 3-lattice $\mathcal{M}\left(\boldsymbol{\delta}_{\sigma}^{0}\right)$ onto the basal plane of $\boldsymbol{e}_{1}^{0}$ and $\boldsymbol{e}_{2}^{0}$ orthogonal to the optic axis $\boldsymbol{e}_{3}^{0}$. We address to James (1987) (also Pitteri and Zanzotto (2002), Pitteri (2002b)) for the construction of the above 3-lattice structure of $\beta$-quartz by means of suitable helices whose projection on the basal plane is shown in the lower-right part of Fig, 4.

The point group $P\left(\boldsymbol{\delta}_{\sigma}^{0}\right)<S O(3)$ of this 3-lattice is the rotational subgroup $\mathcal{H}_{k}$ (see (40)) of the hexagonal holohedry $P\left(\boldsymbol{e}_{a}^{0}\right)$, and is generated for instance by $\boldsymbol{R}_{k}^{\pi / 3}$ and $\boldsymbol{R}_{i}^{\pi}$. The associated crystal class is called hexagonal trapezohedral, is 
denoted by 622 in the International Tables (1996), and is the actual crystal class of $\beta$-quartz, so that the monatomic 3-lattice $\mathcal{M}\left(\boldsymbol{\delta}_{\sigma}^{0}\right)$ of Si atoms gives already a good approximation of the actual (geometric) symmetry of this quartz phase.

Case (38)

The treatment is the same as in Pitteri (2002b) and is not repeated here.

Case (38) 2

We denote by $\beta_{k}^{\pi / 3}$ the submatrix $\beta$ of any element of the $\operatorname{coset}\left[\nu_{k}^{\pi / 3}\right] \in L^{+}\left(\boldsymbol{\delta}_{\sigma}^{0}\right)$ that corresponds to the rotation $\boldsymbol{R}_{k}^{\pi / 3}$ according to (15), etc., $\beta_{\mathbf{1}}$ denoting the 3 by 3 identity. Based on (15) we have

$$
\begin{gathered}
\beta_{\mathbf{1}}=\left(\begin{array}{lll}
1 & 0 & 0 \\
0 & 1 & 0 \\
0 & 0 & 1
\end{array}\right)=\beta_{k}^{\pi}, \quad \beta_{k}^{\pi / 3}=\left(\begin{array}{lll}
0 & 1 & 0 \\
0 & 0 & 1 \\
1 & 0 & 0
\end{array}\right)=\beta_{k}^{4 \pi / 3}, \\
\beta_{k}^{2 \pi / 3}=\left(\begin{array}{lll}
0 & 0 & 1 \\
1 & 0 & 0 \\
0 & 1 & 0
\end{array}\right)=\beta_{k}^{5 \pi / 3}, \quad \beta_{i}^{\pi}=\left(\begin{array}{lll}
0 & 0 & 1 \\
0 & 1 & 0 \\
1 & 0 & 0
\end{array}\right)=\beta_{j}^{\pi}, \\
\beta_{\sqrt{3} i-j}^{\pi}=\left(\begin{array}{lll}
1 & 0 & 0 \\
0 & 0 & 1 \\
0 & 1 & 0
\end{array}\right)=\beta_{i+\sqrt{3} j}^{\pi}, \quad \beta_{i-\sqrt{3} j}^{\pi}=\left(\begin{array}{ccc}
0 & 1 & 0 \\
1 & 0 & 0 \\
0 & 0 & 1
\end{array}\right)=\beta_{\sqrt{3} i+j}^{\pi} .
\end{gathered}
$$

We now analyze the action induced by $(33)_{2}$ on the 6-dimensional space $\mathcal{S}$ of $\left(\varpi_{1}, \varpi_{2}, \varpi_{3}\right)$, with typical element $\left(a_{1}, a_{2}, a_{3}, b_{1}, b_{2}, b_{3}, c_{1}, c_{2}, c_{3}\right)$ subject to the constraints $a_{i}+b_{i}+c_{i}=0, i=1,2,3$, and the related i.i. subspaces. We first consider the linear action of each $Q$ in $G$ on the whole of $\mathbb{R}^{3 n}$ and the corresponding invariant subspaces, then intersect these with $\mathcal{S}$ to determine the desired i.i. subspaces of the latter.

First of all, the linear action of any $Q \in G$ maps to themselves the subspaces

$$
\begin{gathered}
\mathcal{W}_{1}=\left\{\left(0,0, a_{3}, 0,0, b_{3}, 0,0, c_{3}\right)\right\}, \quad \text { and } \\
\mathcal{W}=\left\{\left(a_{1}, a_{2}, 0, b_{1}, b_{2}, 0, c_{1}, c_{2}, 0\right)\right\},
\end{gathered}
$$

of displacement components along the optic axis and along the basal plane, respectively. This is because the hexagonal group $\mathcal{H}_{k}$ has the optic axis and the basal plane as (irreducible) invariant subspaces.

The (reduced) action of $\mathrm{G}$ on $\mathcal{W}_{1}$ is a group of order 6 , and its action on the coordinates $\left(a_{3}, b_{3}, c_{3}\right)$ is generated by the matrices ${ }^{3}$

$$
\left(\begin{array}{ccc}
0 & 0 & -1 \\
0 & -1 & 0 \\
-1 & 0 & 0
\end{array}\right) \approx \boldsymbol{R}_{i}^{\pi} \approx \boldsymbol{R}_{j}^{\pi} \quad \text { and } \quad\left(\begin{array}{ccc}
0 & 0 & 1 \\
1 & 0 & 0 \\
0 & 1 & 0
\end{array}\right) \approx \boldsymbol{R}_{k}^{\frac{\pi}{3}}
$$

\footnotetext{
${ }^{3}$ Here and below $\approx$ means 'represented by' or 'representing'.
} 
and is the symmetry group of an equilateral triangle in $\mathcal{S}$ with vertices at $(1,0,-1),(0,-1,1),(-1,1,0)$. In particular, the action associated to $\boldsymbol{R}_{k}^{\pi}$ is the identity.

Since the only nontrivial invariant subspace of $\mathcal{W}_{1}$ is orthogonal to $\mathcal{S}, \mathcal{W}_{1} \cap \mathcal{S}$ is a 2-dimensional irreducible invariant subspace of $\mathcal{S}$, and consists of monoclinic 3 -lattices, with axis $\boldsymbol{k}$. To within a rotation of the coordinates on $\mathcal{S}$, this reduced problem is the same as the one in item (3) of Case (38) $)_{1}$ in Pitteri (2002b). As there, the bifurcation diagram consists of three unstable transcritical bifurcating curves of orthorhombic 222 symmetry. For instance, the orthorhombic axes (besides $\boldsymbol{k}$ ) are $\boldsymbol{i}$ and $\boldsymbol{j}$ for the choice

$$
\varpi_{1}=\lambda \boldsymbol{k}=-\varpi_{3}, \quad \varpi_{2}=\mathbf{0}, \quad \lambda \in \mathbb{R} .
$$

This corresponds to the 1-dimensional subspace

$$
\left(a_{3}, b_{3}, c_{3}\right)=\gamma(1,0,-1), \quad \gamma \in \mathbb{R}
$$

of $\mathcal{S}$, which is invariant under the identical actions of $\boldsymbol{R}_{i}^{\pi}$ and $\boldsymbol{R}_{j}^{\pi}$.

The subspace $\mathcal{W} \cap \mathcal{S}$ decomposes into the orthogonal sum of three i.i. subspaces, $\mathcal{W}_{2}, \mathcal{W}_{3}, \mathcal{W}_{4}$, the first two of dimension 1 , the third of dimension 2. They are respectively generated by

$$
\begin{gathered}
w_{2}=(1, \sqrt{3}, 0,-2,0,0,1,-\sqrt{3}, 0), \\
w_{3}=(-\sqrt{3}, 1,0,0,-2,0, \sqrt{3}, 1,0), \text { and } \\
w_{4}=\left(\frac{\sqrt{3}}{6},-\frac{1}{2}, 0,-\frac{\sqrt{3}}{3}, 0,0, \frac{\sqrt{3}}{6}, \frac{1}{2}, 0\right), w_{4}^{\prime}=\left(\frac{1}{2}, \frac{\sqrt{3}}{6}, 0,0,-\frac{\sqrt{3}}{3}, 0,-\frac{1}{2}, \frac{\sqrt{3}}{6}, 0\right) .
\end{gathered}
$$

The reduced group on $\mathcal{W}_{2}\left[\mathcal{W}_{3}\right]$ is $\{1,-1\}$; for instance

$$
1 \approx \boldsymbol{R}_{i}^{\pi}\left[1 \approx \boldsymbol{R}_{j}^{\pi}\right] \text { and }-1 \approx \boldsymbol{R}_{k}^{\pi / 3} \approx \boldsymbol{R}_{k}^{\pi}
$$

Therefore, as is known, the bifurcation diagram is the standard pitchfork. A fourth-order polynomial energy is sufficient to capture the qualitative features of a (supercritical) second-order bifurcation, while a subcritical first-order one, as in the case of quartz, requires a sixth-order polynomial (see for instance Ericksen (1993), (2001) or Pitteri and Zanzotto (2002)). In $\mathcal{W}_{2}$ [in $\mathcal{W}_{3}$ ] the crystal class of the bifurcating multilattices is trigonal trapezohedral (32 in the International Tables (1996)), with $\boldsymbol{k}$ as 3-fold axis; the additional generator of the point group is $\boldsymbol{R}_{i}^{\pi}$ [is $\boldsymbol{R}_{j}^{\pi}$ ].

The reduced group on $\mathcal{W}_{4} \cap \mathcal{S}=\left\{x w_{4}+y w_{4}^{\prime}\right\}$ has order 12 , and the changes in the coordinates $x$ and $y$ are generated by the matrices

$$
\left.-f \approx \boldsymbol{R}_{i}^{\pi} \text { (or, equivalently, } f \approx \boldsymbol{R}_{j}^{\pi}\right) \text { and } r(\pi / 3) \approx \boldsymbol{R}_{k}^{\frac{\pi}{3}}
$$

this is the symmetry group of a regular hexagon in $\mathbb{R}^{2}$ with center at the origin and a vertex on the $x$ axis. This reduced problem is the same as the one in item 


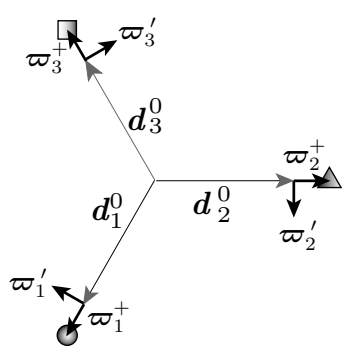

Figure 5: Displacement increments $\varpi_{r}^{+}$and $\varpi_{r}^{\prime}$ producing the two trigonal trapezohedral quartz phases for the same $\lambda>0$

(4) of Case (38) 1 in Pitteri (2002b). As there, the bifurcating branches consist in two triples of symmetry-related pitchforks, all of monoclinic 2 crystal class. Only one of the triples can be stable.

We now analyze in detail the two trigonal trapezohedral subspaces $\mathcal{W}_{2}$ and $\mathcal{W}_{3}$. The reference displacement increments corresponding to $\mathcal{W}_{2}$ are, $\bar{\lambda}, \lambda^{\prime}$ being real parameters,

$$
\begin{gathered}
\varpi_{1}^{+}=\bar{\lambda}(1, \sqrt{3}, 0)=-\lambda^{\prime}\left(\boldsymbol{e}_{1}^{0}+\boldsymbol{e}_{2}^{0}\right), \\
\varpi_{2}^{+}=\bar{\lambda}(-2,0,0)=\lambda^{\prime} \boldsymbol{e}_{1}^{0}, \quad \varpi_{3}^{+}=\bar{\lambda}(1,-\sqrt{3}, 0)=\lambda^{\prime} \boldsymbol{e}_{2}^{0} .
\end{gathered}
$$

Equivalently, by (26), denoting by $\boldsymbol{d}_{r}^{+}$the present shifts, with $\boldsymbol{d}_{r}=\boldsymbol{U} \boldsymbol{d}_{r}^{0}, \boldsymbol{e}_{a}=$ $\boldsymbol{U} \boldsymbol{e}_{a}^{0}$, and $\lambda$ a real parameter,

$$
d_{1}^{+}=d_{1}-\lambda\left(e_{1}+e_{2}\right), \quad d_{2}^{+}=d_{2}+\lambda e_{1}, \quad d_{3}^{+}=d_{3}+\lambda e_{2} .
$$

The displacement increments are directed radially from the center of mass, outwards for $\lambda>0$ (see Fig. 5). These displacements represent deformed $\beta$-quartz for $\lambda=0$, while for $\lambda \neq 0$ they give the $\mathcal{M}\left(\boldsymbol{\delta}_{\sigma}^{+}\right)$3-lattice model for trigonal trapezohedral $\alpha$-quartz proposed in James (1987), and used also in Ericksen (2001) and Pitteri and Zanzotto (2002). Indeed, putting the origin on a point in the lattice displaced by $\boldsymbol{d}_{1}^{+}$, the corresponding shifts are, in obvious notation,

$$
\boldsymbol{p}_{1}^{+}=\boldsymbol{d}_{3}^{+}-\boldsymbol{d}_{1}^{+}=\boldsymbol{p}_{1}+\lambda\left(\boldsymbol{e}_{1}+2 \boldsymbol{e}_{2}\right), \quad \boldsymbol{p}_{2}^{+}=\boldsymbol{d}_{2}^{+}-\boldsymbol{d}_{1}^{+}=\boldsymbol{p}_{2}+\lambda\left(2 \boldsymbol{e}_{1}+\boldsymbol{e}_{2}\right) .
$$

We address to James (1987) (also Pitteri and Zanzotto (2002), Pitteri (2002b)) for a description of how the $\alpha$-quartz structure can be obtained by deforming the helices mentioned above for $\beta$-quartz.

The projection of the $\mathcal{M}\left(\boldsymbol{\delta}_{\sigma}^{+}\right)$3-lattice onto the (basal) plane of $\boldsymbol{e}_{1}$ and $\boldsymbol{e}_{2}$ is sketched in Fig. 6 for $\lambda>0$. For fixed $\lambda$, the other possibility is given by the Dauphiné twin $\mathcal{M}\left(\boldsymbol{\delta}_{\sigma}^{-}\right)$, where the $\boldsymbol{\delta}_{\sigma}^{-}=\left(\boldsymbol{e}_{a}, \boldsymbol{d}_{1}^{-}, \boldsymbol{d}_{2}^{-}, \boldsymbol{d}_{3}^{-}\right)$have the same lattice vectors $\boldsymbol{e}_{a}$ as $\boldsymbol{\delta}_{\sigma}^{+}$, and displacements

$$
d_{1}^{-}=d_{1}+\lambda\left(e_{1}+e_{2}\right), \quad d_{2}^{-}=d_{2}-\lambda e_{1}, \quad d_{3}^{-}=d_{3}-\lambda e_{2},
$$




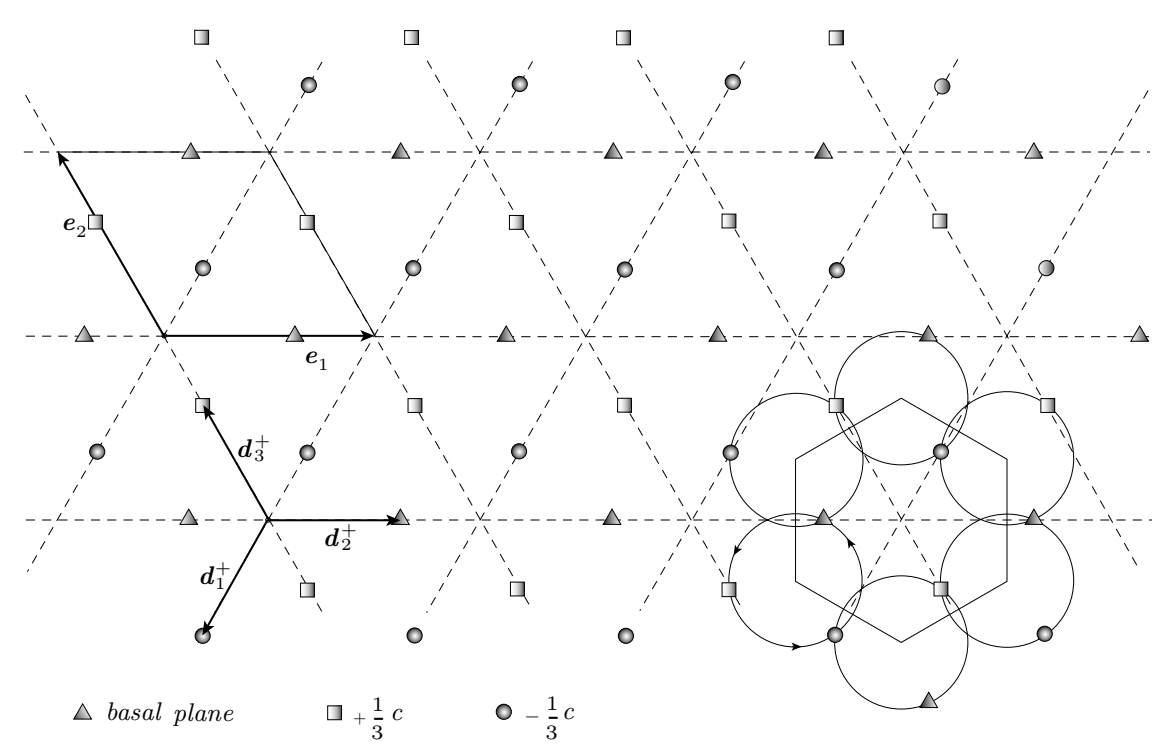

Figure 6: Projection as in Fig. 4 for the right-handed $\alpha$-quartz structure, and of the descriptors $\boldsymbol{\delta}_{\sigma}^{+}=\left(\boldsymbol{e}_{a}, \boldsymbol{d}_{1}^{+}, \boldsymbol{d}_{2}^{+}, \boldsymbol{d}_{3}^{+}\right)$for the 3-lattice given by (39) and (57) with $\boldsymbol{U}=\mathbf{1}$ and $\lambda>0$

with the same $\lambda$ as in (57). Pictorially, in Fig. 6 one has to perform on the displacements $\boldsymbol{d}_{r}^{+}$a rotation $\boldsymbol{R}_{k}^{\pi}$ with respect to the center of mass. These two twinned configurations correspond to symmetry-related points on the bifurcated branches of the pitchfork in the $\mathcal{W}_{2}$ subspace mentioned above. We address the reader to Pitteri and Zanzotto (2002) for more details on Dauphiné twins, and recall that, as hinted at above, the twin multilattice descriptors $\boldsymbol{\delta}_{\sigma}^{-}$can be obtained from $\boldsymbol{\delta}_{\sigma}^{+}$by means of the rotation $\boldsymbol{R}_{k}^{\pi}$, of order 2 (see also (53)).

The reference displacement increments corresponding to elements of the subspace $\mathcal{W}_{3}$ are, in terms of real parameters $\bar{\nu}, \nu^{\prime}$,

$$
\begin{gathered}
\varpi_{1}^{\prime}=\bar{\nu}(-\sqrt{3}, 1,0)=\nu^{\prime}\left(\boldsymbol{e}_{2}^{0}-\boldsymbol{e}_{1}^{0}\right), \\
\varpi_{2}^{\prime}=\bar{\nu}(0,-2,0)=-\nu^{\prime}\left(2 \boldsymbol{e}_{2}^{0}+\boldsymbol{e}_{1}^{0}\right), \quad \varpi_{3}^{\prime}=\bar{\nu}(\sqrt{3}, 1,0)=\nu^{\prime}\left(2 \boldsymbol{e}_{1}^{0}+\boldsymbol{e}_{2}^{0}\right) .
\end{gathered}
$$

Equivalently, by (26), denoting by $\boldsymbol{d}_{r}^{\prime}$ the corresponding present displacements,

$$
\boldsymbol{d}_{1}^{\prime}=\boldsymbol{d}_{1}+\frac{\mu}{3}\left(e_{2}-e_{1}\right), \quad \boldsymbol{d}_{2}^{\prime}=\boldsymbol{d}_{2}-\frac{\mu}{3}\left(2 e_{2}+e_{1}\right), \quad \boldsymbol{d}_{3}^{\prime}=\boldsymbol{d}_{3}+\frac{\mu}{3}\left(2 e_{1}+e_{2}\right),
$$

with $\mu$ a real parameter. The displacement increments are azimuthal, oriented clockwise for $\mu>0$. We have deformed $\beta$-quartz for $\mu=0$, while symmetryrelated points on the bifurcated branches of the pitchfork in this subspace correspond to opposite values of $\mu \neq 0$ in the displacements given by (62). The related multilattices, say $\mathcal{M}\left(\boldsymbol{\delta}_{\sigma}^{\prime}\right)$ and $\mathcal{M}\left(\boldsymbol{\delta}_{\sigma}^{\prime \prime}\right)$, are another example of shuffle 


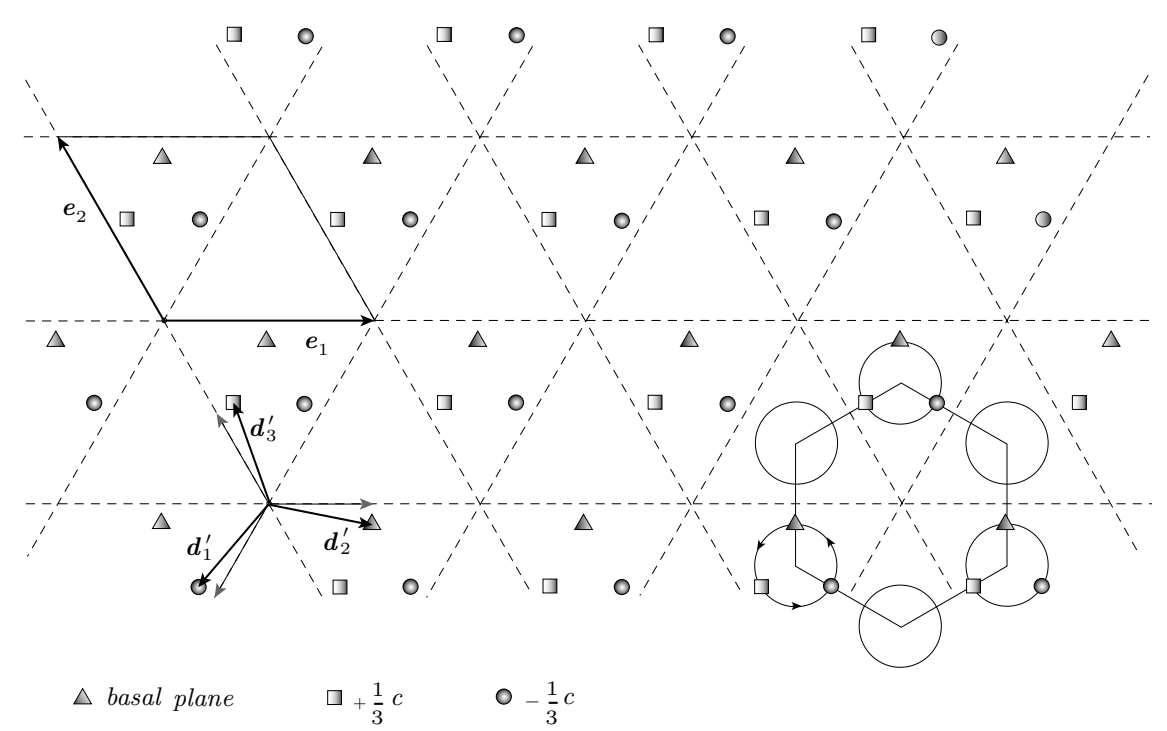

Figure 7: Projection as in Fig. 6 for the right-handed quartz structure with displacements given by (62) for $\boldsymbol{U}=\mathbf{1}$ and $\mu>0$

twins, very similar to the Dauphiné twins described above. In particular, the twin multilattice $\mathcal{M}\left(\boldsymbol{\delta}_{\sigma}^{\prime \prime}\right)$ can be obtained from $\mathcal{M}\left(\boldsymbol{\delta}_{\sigma}^{\prime}\right)$ by means of the same rotation $\boldsymbol{R}_{k}^{\pi}$, of order 2 about the center of mass, which relates the Dauphiné twins (see (53)). This can be obtained pictorially from Figs 5 and 7: by replacing each $\varpi_{r}^{\prime}$ with its negative, the corresponding $\boldsymbol{d}_{r}^{\prime}$ becomes its symmetric with respect to the line of $\boldsymbol{d}_{r}^{0}$, and the symmetric displacement is the $\boldsymbol{R}_{k}^{\pi}$-transform of a displacement equivalent to $\boldsymbol{d}_{r}^{\prime}$; for $r=1$ this is $\boldsymbol{d}_{1}^{\prime}+\boldsymbol{e}_{1}+\boldsymbol{e}_{2}$, etc.

By putting the origin on a point in the lattice displaced by $\boldsymbol{d}_{1}^{\prime}$, we obtain the shifts describing this quartz phase in Pitteri (2002b):

$$
p_{1}^{\prime}=d_{3}^{\prime}-d_{1}^{\prime}=p_{1}+\mu e_{1}, \quad p_{2}^{\prime}=d_{2}^{\prime}-d_{1}^{\prime}=p_{2}-\mu e_{2}
$$

As in Pitteri (2002b) one can describe this low-symmetry phase and the twins in terms of deformation of the reference $\beta$-quartz helices. Now the radius of those helices is shrinked, hence neighboring helices do not intersect anymore. Fig. 7 shows one of the possible arrangements of the actual helices. Looking at the hexagon drawn in the lower right corner, the other possibility - which gives the twinned configuration - is obtained by exchanging the occupied and the nonoccupied helices in that hexagon, and then coherently in the whole structure.

We address the reader to Pitteri (2002b) for some detail of how the trigonal subspaces above enter the kinematics on which Ericksen (2001) bases his bifurcation analysis of the $\alpha-\beta$ transition. 


\section{References}

Budiansky, B. and L. Truskinovsky (1993) On the mechanics of stress-induced phase transformations in zirconia, J. Mech. Phys. Solids, 41, 1445-1459.

Ericksen, J. L. (1993) Local bifurcation theory for thermoelastic Bravais lattices. In: J.L. Ericksen, R.D. James, D. Kinderlehrer and M. Luskin (eds.) Microstructure and phase transition, Vol. 54 of the IMA Volumes in Mathematics and its Applications, Springer-Verlag, New York, etc.

Ericksen, J. L. (2001) On the theory of the $\alpha-\beta$ phase transition in quartz, $J$. of Elasticity, 63, 61-86.

Fadda, G., L. Truskinovsky and G. Zanzotto (2002) Unified Landau description of the tetragonal, orthorhombic, and monoclinic phases of zirconia, Phys. Rev. $B, 66,174107$ 1-10.

Golubitsky, M., D. Schaeffer and I. Stewart (1988) Singularities and groups in bifurcation theory, Vol II. Springer Verlag, New York, etc. Applied Mathematical Sciences vol.69.

International Tables (1996) International Tables for X-ray Crystallography, Volume A. Reidel Publishing Company, Dordrecht, Boston. T. Hahn (Ed.).

James, R. D. (1987) The stability and metastability of quartz. In: Antman, S. S., J. L. Ericksen, D. Kinderlehrer and I. Müller (eds.) Metastability and Incompletely Posed Problems, Vol. 3 of the IMA Volumes in Mathematics and its Applications, Springer-Verlag, New York, etc.

Pitteri, M. (2002a) On bifurcations in multilattices. In: Monaco, R., M. Pandolfi and S. Rionero (eds.) Proceedings of WASCOM 2001, Porto Ercole, Italy. World Scientific, Singapore etc.

Pitteri, M. (2002b) On weak phase transformations in multilattices. Preprint Series, n. 100, TMR network "Phase Transitions in Crystalline Solids". Also Rapporto Tecnico DMMMSA n. 88, 2/12/2002; appearing in J. of Elasticity.

Pitteri, M. and G. Zanzotto (2002) Continuum models for phase transitions and twinning in crystals. CRC/Chapman \& Hall, Boca Raton, London, etc.

Simha, N. K. and L. Truskinovsky (1996) Phase diagram of zirconia in stress space. In: Batra, R. and M. Beatty (eds.) Contemporary research in the mechanics and mathematics of materials, CIMNE, Barcelona.

Tolédano, P. and V. Dmitriev (1996) Reconstructive phase transformations: in crystals and quasicrystals. World Scientific, Singapore etc. 